\title{
Solución con calidad y reducción de tiempo clínico en prótesis bucal. Reporte de un caso
}

\section{Solution with quality and reduction of clinical procedure in the oral rehabilitation. Case report}

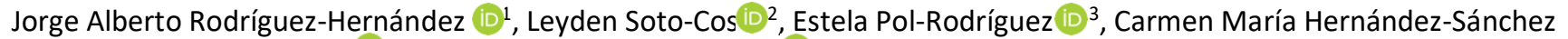 \\ (iD) 4 , Mayra de la C. Pérez-Álvarez (D) ${ }^{5}$, Maydel Pérez-Fuentes (iD) ${ }^{6}$.
}

1. Clínica Estomatológica Docente de Bauta. Artemisa, Cuba. Correo: rodalba@infomed.sld.cu - https://orcid.org/0000-0002-0169-8493

2. Universidad Victoria de Girón. La Habana, Cuba. Correo: leydensoto@infomed.sld.cu - https://orcid.org/0000-0002-1520-4087

3. Centro de Estomatología de la Clínica Central Cira García. La Habana, Cuba. Correo: estelapol@infomed.sld.cu - https://orcid.org/0000-0001-77198922

4. Centro de Estomatología de la Clínica Central Cira García. La Habana, Cuba. Correo: carmenhdez@infomed.sld.cu - https://orcid.org/0000-00024065-4442

5. Clínica Estomatológica Docente de Bauta, Artemisa. Universidad de la Habana. La Habana, Cuba. Correo: mayraperezcuba61@gmail.com https://orcid.org/0000-0003-2384-9122

6. Universidad de Ciencias Médicas de La Habana. La Habana, Cuba. Correo: maydelpf@infomed.sld.cu - https://orcid.org/0000-0001-7437-8792 tiempo clínico en prótesis bucal. Reporte de un caso. Duazary. 2020 octubre; 17(4): 89-95. Doi: https://doi.org/10.21676/2389783X.3604

\section{RESUMEN}

Palabras clave: prótesis dentaria inmediata; extracciones múltiples; rehabilitación protésica.
En ocasiones, a los pacientes que precisan rehabilitaciones extensas se les dificulta el tratamiento por el tiempo y por visitas repetidas a la institución de salud. Se reporta un caso clínico con estas características, que presentaba considerable pérdida ósea en todos los dientes remanentes por lesiones periodontales graves y caries dental, necesitado de extracciones dentarias múltiples en cirugía bucal y rehabilitación protésica total de forma inmediata, pues disponía de limitado tiempo para tratarse. Al paciente no le era posible rehabilitar con implantes dentarios bajo estas condiciones, de manera que se le indicó prótesis inmediata, agrupando etapas del tratamiento, para disminuir el tiempo del procedimiento habitual sin descuidar la calidad. Se logró un trabajo acorde a las exigencias estético-funcionales del paciente agrupando los pasos de la cirugía bucal y con la confección e instalación de prótesis inmediata en tiempo reducido.

\section{ABSTRACT}

Keywords: immediate dental prosthesis; multiple extractions; prosthetic rehabilitation.
Patients who require extensive rehabilitation are sometimes difficult to treat due to time and repeated visits to the health institution. We report a clinical case with these characteristics, which presented, in all the remaining teeth, considerable bone loss due to serious periodontal injuries and dental caries, in need of multiple dental extractions in oral surgery and rapid total prosthetic rehabilitation immediately, as there was limited time to treat. The patient could not be rehabilitated with dental implants under these conditions. To achieve this purpose, an immediate prosthesis was indicated, where treatment stages are grouped, to reduce the time of the usual procedure, without neglecting quality. A work was carried out according to the aesthetic-functional demands of the patient, grouping the steps of oral surgery and the preparation and installation of an immediate prosthesis, in a reduced time. 


\section{INTRODUCCIÓN}

Algunos pacientes rechazan dedicar tiempo y consultas para realizarse tratamientos estomatológicos, lo que trae consigo un deterioro paulatino de la salud bucal, provocando lesiones incurables de dientes que requieren extracciones y rehabilitación del daño. La prótesis dental es una necesidad asistencial que no puede considerarse como un problema aislado pues compromete la calidad de vida por la limitación funcional y estética de la pérdida dentaria ${ }^{1,2}$.

La ausencia de dientes se traduce en reabsorción o atrofia del hueso alveolar, que constituye una entidad crónica, progresiva, irreversible y acumulativa que da lugar a una paulatina transformación de la morfología facial ${ }^{3,4}$. Cuando la causa de ausencia dentaria es caries, por lo regular la altura ósea disminuye de forma menos agresiva, mientras que en enfermedad periodontal (específicamente, periodontitis) se manifiesta una destrucción avanzada del hueso alveolar ${ }^{5-7}$.

En muchos casos, cuando el paciente no quiere someterse a cirugía de rehabilitación ósea, la mejor alternativa de tratamiento es la prótesis inmediata. Esta se aplica cuando la integridad anatómica de los dientes que se van sustituir permite guiarse para seleccionar las piezas dentales artificiales, o cuando el paciente es incapaz de tolerar la ausencia de dientes antes de colocarse una prótesis. Otra condición para recurrir a este tratamiento es que el paciente a la vez sea capaz de soportar un aparato protésico sobre los tejidos lacerados por las extracciones recientes. Además, las prótesis inmediatas evitan el periodo de no tener dientes naturales o artificiales, siendo el resultado estético un tema trascendental dentro de la rehabilitación ${ }^{4,8,9}$.

La prótesis inmediata se define como una prótesis total o parcial fabricada para colocarla tan pronto se retiran los dientes naturales ${ }^{10}$. Las dentaduras inmediatas se construyen antes de extraer los dientes remanentes y les permiten a los pacientes continuar con sus actividades sociales y de negocios sin pasar por un estado edéntulo. Este medio de rehabilitación se coloca sobre los rebordes alveolares tratados y le permite al hueso alveolar remodelarse y ajustarse al aditamento protésico ${ }^{8,10,11}$.

El paciente del caso reportado en este artículo fue tratado en el Centro Estomatológico de la Clínica Internacional Cira García, que posee entre su colectivo de profesionales a profesores de la universidad que aplican sus conocimientos e iniciativas para mejorar la calidad de la atención. El objetivo planteado aquí es dar cuenta de un caso clínico con enfermedad periodontal avanzada, tributario de extracciones múltiples con cirugía bucal y colocación de la prótesis inmediata estética y funcional en solo siete días.

\section{REPORTE DE CASO CLÍNICO}

Se recibió al paciente ANB, de 63 años y procedente de Bahamas, con buen estado de salud general pero aquejado de enfermedad periodontal con bolsas $\geq 8$ $\mathrm{mm}$ de profundidad al sondaje. La pérdida ósea radiográfica se extendía hasta el tercio apical en los dientes remanentes y se observaron $\geq 5$ pérdidas dentarias por razones periodontales, disfunción masticatoria, trauma oclusal secundario y movilidad dentaria $\geq 2$, con defecto de cresta grave que se enmarca en estadio IV de acuerdo a la nueva clasificación de las enfermedades periodontales ${ }^{12,13}$. También se registró caries dental con criterios 4 y 6 en la clasificación ICDAS II, lo que define la situación precaria de los dientes ${ }^{14}$.

El paciente buscaba una solución mediante un tratamiento eficaz y de corto tiempo, pues solo contaba con siete días. Se procedió entonces a establecer diagnóstico, pronóstico y plan de tratamiento protésico-quirúrgico para instalar prótesis inmediata en cuatro sesiones de trabajo, en lugar de las siete establecidas para el protocolo de confección de una prótesis total inmediata.

La mayor parte de procedimientos clínicos y de laboratorio para este tipo de prótesis son similares a los correspondientes a las prótesis totales convencionales; solo se diferencian en que las inmediatas se construyen antes de extraer los 
dientes remanentes y se colocan justo después de extraerlos. La prótesis total inmediata es una buena alternativa de tratamiento en pacientes que requieren la extracción de todos sus dientes por motivos de enfermedad periodontal y caries, que les permite hacer una transición menos traumática y obtener una adecuada estética y confort ${ }^{10}$.

La primera sesión comenzó con información al paciente sobre los cambios al tratamiento convencional en cuanto a sesiones y frecuencias del tratamiento. Una vez entendido y firmado el consentimiento informado por el implicado, se ejecutaron los pasos previstos de confección de historia clínica $(\mathrm{HC})$, toma de impresiones con alginato para modelos de estudio y determinación de la relación cráneo-mandibular, incluida toma de dimensión vertical de reposo y oclusiva.

En el laboratorio el protesista realizó la preparación quirúrgica del modelo teniendo en cuenta las condiciones óseas, el grado de reabsorción de la cresta, la existencia de bolsas periodontales y su profundidad, así como el régimen de reabsorción del maxilar y la mandíbula. Para valorar estos factores se tomaron las radiografías periapicales de los dientes que se iban a extraer y se observaron detalladamente. En la preparación de las prótesis se utilizaron los propios modelos primarios, recortando el yeso simulando rebordes operados. En el laboratorio y sobre estas piezas, también se confeccionaron las plantillas quirúrgicas transparentes con el estampador del laboratorio (Figura 1).

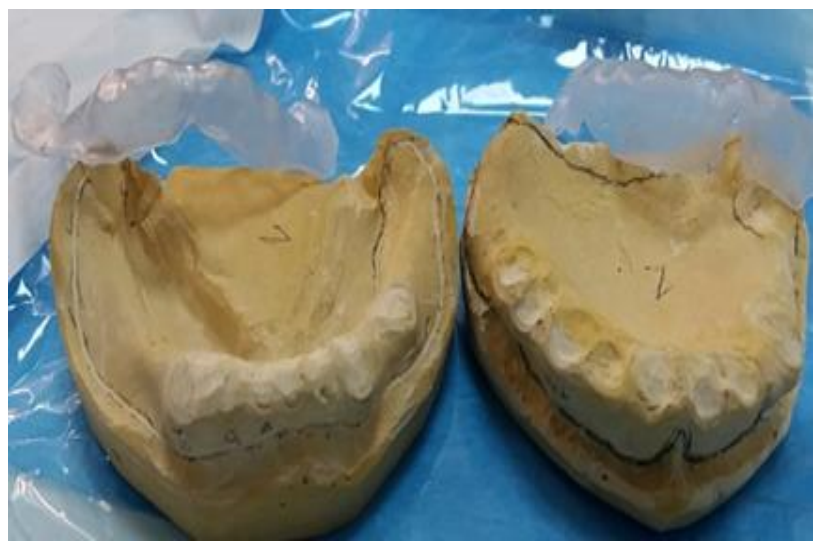

Figura 1. Modelo simulando rebordes operados; encima, las plantillas transparentes.
En la segunda sesión el paciente fue intervenido quirúrgicamente, realizándose anestesia y antisepsia del campo operatorio, extracciones dentarias y regularización ajustada a las plantillas previamente esterilizadas en solución de Hibitane al $2 \%$. Las plantillas se duplicaron con la finalidad de que el paciente permaneciera con las duplicadas en su boca como protector quirúrgico.

Concluida la intervención, las plantillas fueron reutilizadas, previa esterilización similar a la anterior, y sobre ellas se confeccionaron los rollos de articulación con el fin de realizar la relación cráneo-mandibular y su montaje en el articulador para la colocación de los dientes artificiales (Figura 2).

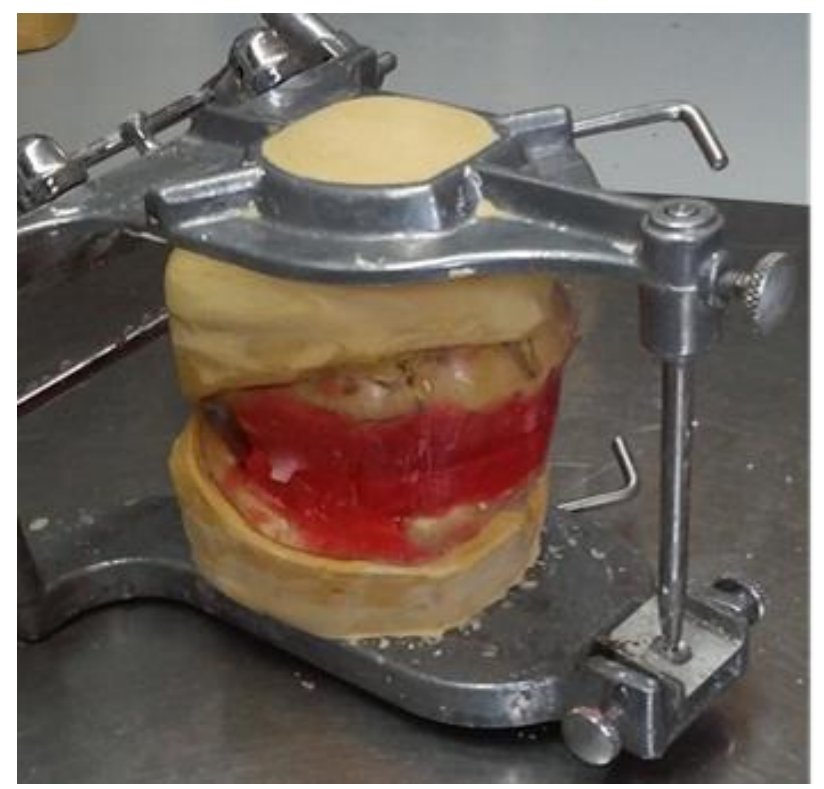

Figura 2. Rollos sobre plantilla quirúrgica montados en el articulador.

En esta misma sesión se articularon los dientes en las propias placas de registro plástico y en el articulador. Luego se procedió a probar el caso y a tomar, con las placas de prueba, la impresión definitiva a boca cerrada con pasta zinquenólica, tal como se muestra en la figura 3.

Vaciados los modelos de las impresiones definitivas, estas se limpiaron muy bien de los restos de pasta zinquenólica, se le realizaron algunos retoques al encerado de terminación y las piezas se colocaron en muflas para continuar normalmente la 
finalización de las prótesis en el laboratorio (Figura. 4).

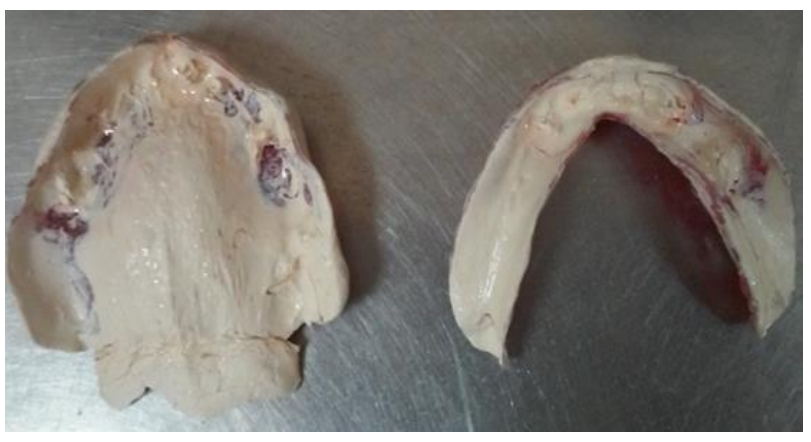

Figura 3. Interior de la impresión definitiva sobre la plantilla con dientes montados.

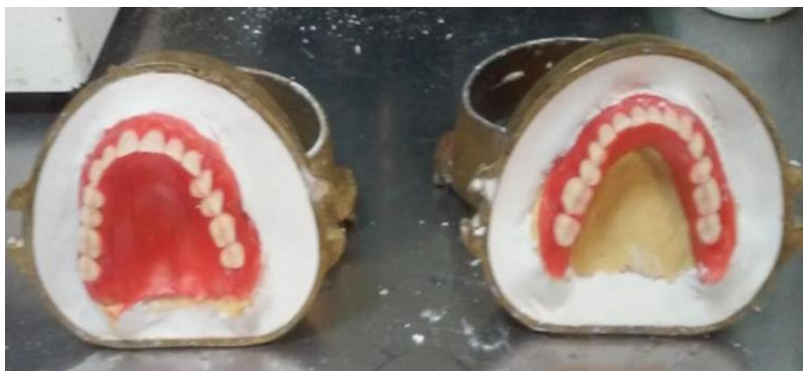

Figura 4. Enmuflado.

Posteriormente se continuó todo el proceso de desenmuflado, descerado, empaquetamiento, cocinado, rebajado y pulido de las prótesis terminadas en acrílico (Figura 5). En un siguiente momento, una vez concluidos los trabajos de laboratorio, se instaló el aditamento protésico en la clínica (Figura 6) y se comprobó que existían todos los parámetros clínicos de retención, estabilidad y sellado periférico, entre otros. El paciente se manifestó con una opinión de conformidad y satisfacción por cumplir con las expectativas previstas, pues se restablecieron eficazmente la fonación, la risa, el bostezo y la masticación. A la vez, este tipo de prótesis logrará en el tiempo el remodelado óseo del reborde alveolar, adaptándose a las bases acrílicas de la prótesis inmediata.

De este modo, se describe una modificación en el tiempo y las sesiones de trabajo para la confección de una prótesis total inmediata en cuatro visitas clínicas, incluyendo la cirugía. Realizar esta modificación no requiere de habilidades especiales; solo necesita agrupar pasos, que se realizaron en coordinación entre los profesionales de cirugía oral, laboratorio de prótesis y actividad clínica protésica. Esta técnica, además, permite ahorrar placas de base ideal para cubetas y para placas de articulación y la cantidad de yeso en el duplicado de modelos. El tiempo de estancia del paciente en la institución y la disminución de consultas son ventajas que se logran aplicando estas variantes de tratamiento.

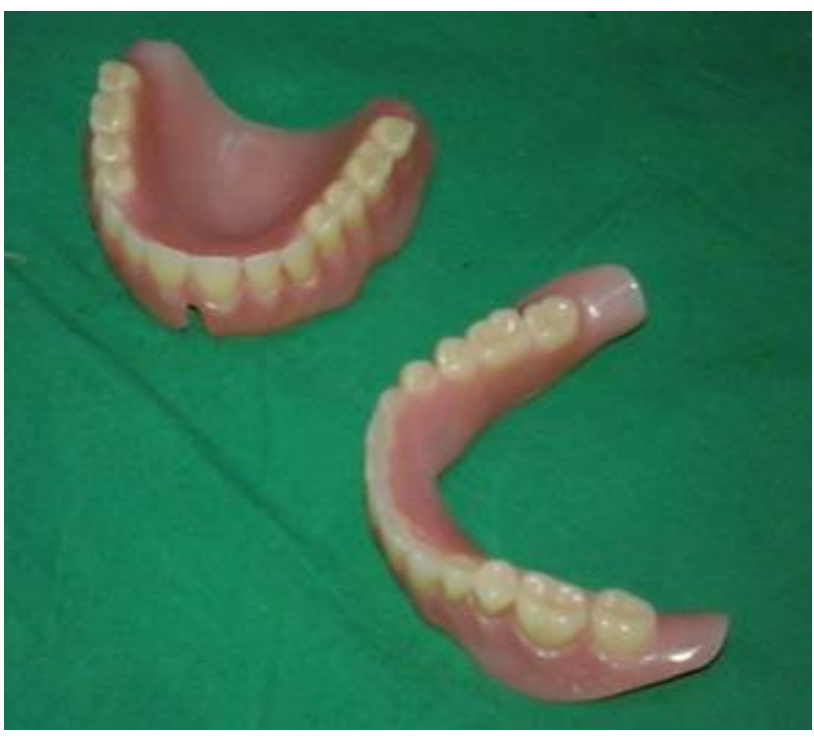

Figura 5. Prótesis terminadas en acrílico.

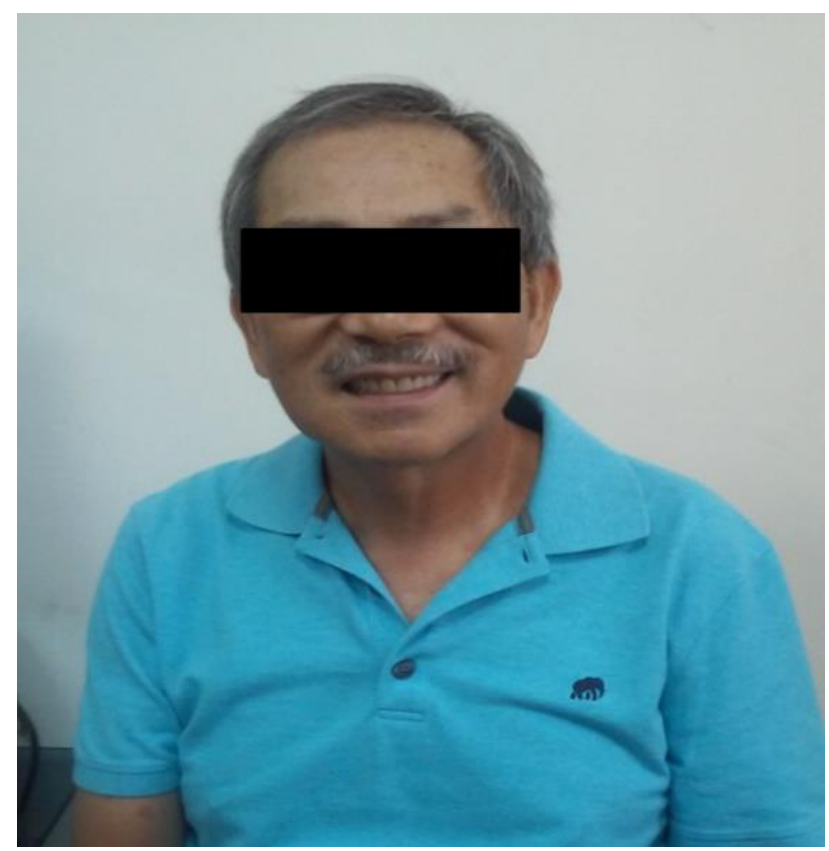

Figura 6. Instalación de la prótesis.

\section{Declaración sobre aspectos éticos}


Para realizar todo el tratamiento se le respetó al paciente la Declaración de Helsinki ${ }^{15}$, informándole sobre los objetivos y las particularidades del procedimiento y solicitando por escrito su consentimiento de participación.

\section{DISCUSIÓN}

El resultado estético en la rehabilitación protésica es un tema trascendental que influye en el comportamiento psicosocial y en la calidad de vida general del paciente. Al igual que en este trabajo, Orrego et $a l^{4}$ resaltan la importancia de definir los objetivos de tratamiento en conjunto y lograr una atención acorde a las necesidades de cada persona que acude al profesional que trabaja sobre estética.

Así mismo, similar a lo reportado en este caso clínico, Fuertes et $a l^{15}$ describieron los pasos clínicos y de laboratorio en la rehabilitación con prótesis inmediata y mostraron también el éxito de la cicatrización y la mínima reabsorción del reborde, lo que representa ventajas estéticas, biológicas, quirúrgicas, masticatorias y fonéticas. Criado et $a l^{16}$ mostraron igualmente muy buenos resultados estéticos, funcionales y psíquicos en el tratamiento de rehabilitación con prótesis inmediata luego de una amplia recesión quirúrgica.

Valladares ${ }^{17}$ también realizó una modificación en el proceder de la técnica en cuanto a la preparación quirúrgica del modelo de trabajo y la ubicación de los dientes artificiales para permitir la prueba de las zonas edentes. De esta forma se comprueban mediante la placa las relaciones ocluso-articulares, dimensión vertical y estética. El $100 \%$ de los pacientes presentaron satisfacción estética, por lo que el montaje de los dientes se realizó de una sola vez, reduciendo el tiempo necesario para el desarrollo de este paso. Corona et $a^{18}$, por su parte, plantearon un similar tratamiento como beneficioso, ya que la cicatrización se orienta y el remodelado óseo mejora.

Con análogos criterios a los manifestados en este trabajo, Vinent et al ${ }^{19}$ comentan que el éxito de la rehabilitación inmediata está dado fundamentalmente por la correcta planificación, la evaluación integral del área donde se instalará el aparato, el diseño y la construcción adecuada de este, lo que evitará incompatibilidades e inadaptaciones a la prótesis.

\section{CONCLUSIÓN}

Con la técnica descrita se logró la rehabilitación protésica del paciente con enfermedad periodontal avanzada, de forma estética y funcional, mediante extracciones múltiples en cirugía bucal y colocación de la prótesis inmediata. La intervención quirúrgicaprotésica fue realizada agrupando etapas del tratamiento para disminuir tiempo del procedimiento habitual, sin descuidar la calidad.

\section{DECLARACIÓN SOBRE CONFLICTOS DE INTERESES}

No existen entre los autores de esta publicación, ni entre las instituciones $y / o$ productos que se mencionan en el manuscrito, conflictos de interés que declarar.

\section{CONTRIBUCIÓN DE LOS AUTORES}

Primer autor: trabajo clínico y redacción.

Segundo autor: trabajo clínico.

Tercero y cuarto autor: trabajo de laboratorio.

Quinto autor: diseño quirúrgico, redacción y envíos.

Sexto autor: diseño metodológico y búsqueda bibliográfica.

\section{REFERENCIAS BIBLIOGRÁFICAS}

1. Corona Carpio MH, Rey Prado B, Arias Arañó Z, Núñez Antúnez L. Manual instructivo de instalación y control de prótesis totales. Rev. Cubana Estomatol. [revista en la Internet]. 2007 Dic [citado 2019 Mar 26]; 44(4). Disponible en: http://scielo.sld.cu/scielo.php?script=sci_arttext\&p id=S0034-75072007000400013\&lng=es.g

2. Jiménez Quintana Z, Justo Díaz $M$, Quesada Peña $S$, Ortiz Palacios Y. Influencia del edentulismo en la calidad de vida del adulto mayor. Convención de Salud [revista en la Internet]. 2018 [citado 2019 Mar 26]; $528 . \quad$ Disponible en: http://www.convencionsalud2018.sld.cu/index.php /connvencionsalud/2018/schedConf/presentations 
3. Guerra Cobian O, Sánchez Silot $C$, Hernández Pedroso L, Felipe Torres S. Efectividad de técnicas de preservación alveolar para rehabilitaciones protésicas e implantoprotésicas. Revista Habanera de Ciencias Médicas [revista en la Internet]. 2018 [citado 2019 Mar 26]; 17(2): 10-20. Disponible en: http://www.revhabanera.sld.cu/index.php/rhab/ar ticle/view/1986

4. Orrego-Ramírez C, Meza-Fuentealba C, VergaraNúñez C, Lee-Muñoz Ximena, Schleyer-Daza N. The aesthetic self-perception of the edentulous patient compared to the opinion of experts. Rev. Clin. Periodoncia Implantol. Rehabil. Oral [Internet]. 2016 Abr [citado 2019 Mar 26]; 9(1): 54-58. Disponible en: https://scielo.conicyt.cl/pdf/piro/v9n1/art10.pdf

5. American Academy of Periodontology. Parameter on chronic periodontitis with slight to moderate loss of periodontal support. J. Periodontol. 2000; 71: 853-855.

6. American Academy of Periodontology. Parameter on chronic periodontitis with advanced loss of periodontal support. J. Periodontol. 2000; 71: 856858.

7. David Herrera J M, Stefan Renvert LJ. Libro Blanco sobre prevención y tratamiento de las enfermedades periodontales para la salud bucodental y la salud general. Equipo de trabajo de la FDI para el Proyecto Mundial de Salud Periodontal. Disponible en:

https://www.fdiworlddental.org/sites/default/files /media/resources/gphp-2018-white_paper-es.pdf

8. Suárez González MD, Fontaine Machado O, Gutiérrez Olives Y. Rehabilitación con prótesis inmediata. Revista Electrónica Dr. Zoilo E. Marinello Vidaurreta [revista en la Internet]. 2015 [citado 2019 Mar 26]; 40(9): 7-12 Disponible en: http://revzoilomarinello.sld.cu/index.php/zmv/arti cle/view/319

9. Reséndiz Melgar H. Bernal Arciniega R. De las prótesis inmediatas a la sobredentadura: Reporte de un caso. Rev. Odont. Mex [revista en la Internet]. 2014 Dic [citado 2019 Mar 26]; 18(4): 241-248. Disponible http://www.scielo.org.mx/scielo.php?script=sci_art text\&pid=S1870-199X2014000400005\&Ing=es

10. Díaz Torreblanca F, Quintana Del Solar M. Prótesis Total inmediata como alternativa de tratamiento. Reporte de Caso. Rev. Estomatol. Herediana. 2013 Ene-Mar 23; (1): 29-33. Disponible en

https://www.academia.edu/9444426/Pr\%C3\%B3te sis_Total_inmediata_como_alternativa_de_tratami ento

11. Zerón A. La nueva clasificación de enfermedades periodontales. Revista ADM. 2018; 75(3): 122-124. Disponible en www.medigraphic.com/adm https://www.medigraphic.com/cgibin/new/resumen.cgi?IDARTICULO=5525

12. Herrera D, Figuero E, Shapira L, Jin L, Sanz M. La nueva clasificación de las enfermedades periodontales y periimplantarias. Revista científica de la Sociedad Española de Periodoncia. 2018; 1(9); 94-110. Disponible en: http://www.sepa.es/web_update/wpcontent/uploads/2018/10/p11ok.pdf

13. Cerón-Bastidas XA. El sistema ICDAS como método complementario para el diagnóstico de caries dental. Rev. CES Odont. 2015; 28(2): 100-109.

14. Declaración de Helsinki de la AMM - Principios éticos para las investigaciones médicas en seres humanos. 64a Asamblea General. Fortaleza, Brasil; octubre 2013.

15. Fuertes Rufin L, Del Valle Zelenenko O, Justo Díaz M. Rehabilitación con prótesis inmediata en pacientes geriátricos: (presentación de un caso). Rev. Habanera de Ciencias Médicas [revista en la Internet]. 2007 [citado 2019 May 23]; 6(2). Disponible en: http://scielo.sld.cu/scielo.php?script=sci_arttext\&p id=s1729-519x2007000200011\&lng=es

16. Criado Mora ZM, Sarracent H, Almagro Urrutia Z, García Martínez I, Aragón Marino T, Ortiz F. Prótesis inmediata post alveolectomía correctora y tuberoplastia bilateral a propósito de un caso 
clínico. Revista Habanera de Ciencias Médicas. 2012;

11(4): 556-562.

17. Valladares García H. Prótesis inmediata. Valoración clínica de la modificación de la técnica tradicional. Rev. Médica Electrónica [revista en la Internet]. 2014 [citado 2019 May 20]; 25(3): 3-6. Disponible en: http://www.revmedicaelectronica.sld.cu/index.php $/$ rme/article/view/91

18. Corona Carpio MH, Arias Arañó Z, Díaz Corral I. Prótesis inmediata con analgesia acupuntural en una adulta mayor. Medisan. 2016; 20(8); 2018-2022. Disponible en:

http://scielo.sld.cu/scielo.php?pid=S102930192016000800016\&script=sci_arttext\&tlng=en

19. Vinent Céspedes AM, La O Salas N. Importancia estética de la prótesis inmediata Medisan. 2018; 22(2): 200-207. 